\title{
Towards Millijoule Narrowband Terahertz Pulses Using the Chirp-and-Delay Technique
}

\author{
Spencer W. Jolly ${ }^{1,2}$, Frederike Ahr ${ }^{3,4}$, Nicholas H. Matlis ${ }^{3}$, Vincent Leroux ${ }^{1,2}$, Timo Eichner ${ }^{1}$, Koustuban \\ Ravi $^{5}$, Hideki Ishizuki ${ }^{6}$, Takunori Taira ${ }^{6}$, Franz X. Kärtner ${ }^{3,4,5}$, Andreas R. Maier ${ }^{1}$ \\ ${ }^{1}$ Center for Free-Electron Laser Science \& Department of Physics, Universität Hamburg, Luruper Chaussee 149, 22761 Hamburg, Germany \\ ${ }^{2}$ Institue of Physics of the ASCR, ELI-Beamlines project, Na Slovance 2, 18221 Prague, Czech Republic \\ ${ }^{3}$ Deutsches Elektronen Synchrotron (DESY) \& Center for Free-Electron Laser Science, Notkestrasse 85, 22607 Hamburg, Germany \\ ${ }^{4}$ Department of Physics, Universität Hamburg, Luruper Chaussee 149, 22761 Hamburg, Germany \\ ${ }^{5}$ Research Laboratory of Electronics, Massachusetts Institute of Technology, 77 Massachusetts Avenue, Cambridge, MA 02139, USA \\ ${ }^{6}$ Laser Research Center, Institute for Molecular Science, 38 Nishigonaka, Myodaiji, Okazaki 444-8585, Japan \\ Author e-mail address: spencer.jolly@desy.de
}

\begin{abstract}
We show generation of $\mathrm{THz}$ pulses of combined energy above $0.5 \mathrm{~mJ}$ at $0.361 \mathrm{THz}$ using the chirp-and-delay technique.
\end{abstract}

OCIS codes: (190.0190) nonlinear optics; (260.3090) Infrared, far; (160.3730) Lithium niobate

There has been a surge in development of terahertz (THz) sources of high-energy and high-peak-field for applications in linear and nonlinear spectroscopy [1], control of matter [2], and compact electron acceleration [3], for which narrowband pulses are of particular interest. Difference frequency generation (DFG) is the most common method to access these $\mathrm{THz}$ frequencies using near-infrared (NIR) drivers.

High conversion efficiencies require high incident intensity and phase matching of the nonlinear process. Quasiphase-matching (QPM) in periodically poled lithium niobate (PPLN) is a proven candidate for narrowband $\mathrm{THz}$ generation. Pumping with $\sim 100$ fs-duration NIR pulses and cryogenic cooling achieved micro-Joule THz energies via intra-pulse DFG [4], but such short pump pulses are limited by optical damage and nonlinear effects. Chirping the NIR pump and overlapping delayed pulses avoids the optical damage issue (to scale up the pump energy) while still providing the required DFG spectral content. This concept was shown to produce up to $10 \mu \mathrm{J}$ via a tilted pulsefront in bulk lithium niobate [5] and energies of up to $40 \mu \mathrm{J}$ using chirp-and-delay in PPLN [6]. Here, we demonstrate chirp-and-delay in PPLN with large aperture crystals, high input energy, and higher-order spectral phase compensation showing an increase in output energy.

Assuming only a linear chirp the optimal time delay between two chirped pulses is dependent only on the chirp-rate and the desired THz frequency. This was confirmed recently [6]. However, these same results showed efficiencies at the $0.1 \%$ level, still among the highest measured for narrowband THz generation, but also below theoretical predictions up to the $1 \%$ level [7]. The effect of the third-order phase present on the NIR pump pulses is significant for the range present in most high power laser systems, and the laser driving these experiments. Schematically this can be seen in Figure 1, where the curved instantaneous frequency means that across the overlapped chirped pulses there is a varying difference frequency. This has a variety of implications, including decreased conversion efficiency, and more complex interference effects of generated $\mathrm{THz}$ depending on the precise scenario [8].

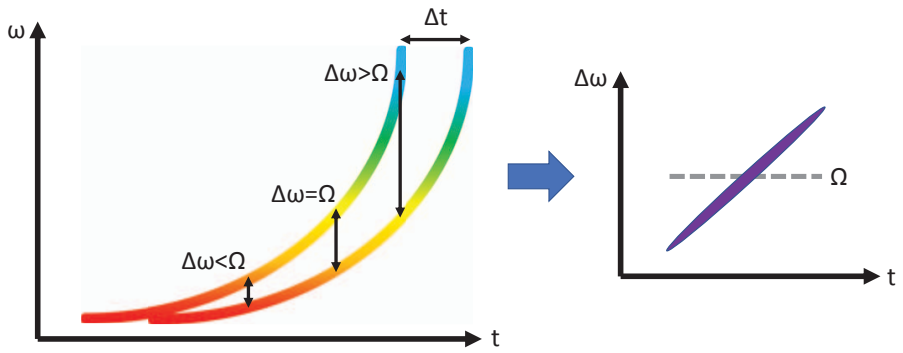

Fig. 1. Cartoon showing the effect of the higher order phase. The curvature of the instantaneous angular frequency $\omega$ of each pulse and the difference frequency $\Delta \omega$ between the overlapped pulse copies results in incomplete matching to the desired THz frequency $\Omega$. 
Experiments were done using uncompressed pulses from the ANGUS system, which has a bandwidth up to $35 \mathrm{~nm}$ FWHM around $800 \mathrm{~nm}$ central wavelength. A pair of pulses was created using a setup similar to a Mach-Zehnder interferometer, with a delay stage in one arm to allow for a tunable relative delay between the two pulses. Due to the Mach-Zehnder topology, there are two outputs of the setup with equal energy and identical temporal properties. We used crystals of two different poling periods $(\Lambda)$ cooled to $80 \mathrm{~K}: \Lambda=212$ and $330 \mu \mathrm{m}$, both with $10 \times 15 \mathrm{~mm}^{2}$ aperture and $36 \mathrm{~mm}$ length [9]. To measure the THz pulse energy a single lens was used to focus onto a pyroelectric detector, and a teflon plate was used to dump the NIR pump and to measure the transmitted optical spectra.

The use of a Mach-Zehnder-like setup allows access to each of the delayed pulses individually. Via this access and manipulation of the phase of each of the overlapped pulses we were able to show an increase of the efficiency and output $\mathrm{THz}$ energy. When pumped up to a fluence up to $399 \mathrm{~mJ} / \mathrm{cm}^{2}(519 \mathrm{~mJ})$ we produced $\mathrm{THz}$ pulses with peak energy above $400 \mu \mathrm{J}$ at $361 \mathrm{GHz}$ frequency (with $1 \%$ bandwidth) in the $330 \mu \mathrm{m}$ poling period PPLN. Using a second identical crystal simultaneously a total average THz energy above $500 \mu \mathrm{J}$ was generated using both outputs of the setup. Results also include pulses above $100 \mu \mathrm{J}$ at $558 \mathrm{GHz}$ frequency from a single $212 \mu \mathrm{m}$ poling period PPLN, pumped at a lower fluence.

These are important steps towards generation of $\mathrm{mJ} \mathrm{THz} \mathrm{pulses.} \mathrm{We} \mathrm{are} \mathrm{potentially} \mathrm{still} \mathrm{limited} \mathrm{by} \mathrm{remaining} \mathrm{higher}$ order dispersion on the pump pulses, and especially saturation as input fluence increases, which when eliminated could significantly increase efficiency, and especially peak $\mathrm{THz}$ energy needed for accelerator applications. Engineering challenges include increasing the performance of the output coupling, and producing an output from more than two crystals at once. These near-term possibilities will push past the mJ barrier and beyond.

\section{References}

1. J. Hebling, K. L. Yeh, M. C. Hoffmann, and K. A. Nelson, "High-power THz generation, THz nonlinear optics, and THz nonlinear spectroscopy," IEEE Journal of Selected Topics in Quantum Electronics 14, 345 (2008).

2. T. Kampfrath, K. Tanaka, and K. Nelson, "Resonant and nonresonant control over matter and light by intense terahertz transients," Nature Photonics 7, 680-690 (2013).

3. E. A. Nanni, W. R. Huang, K.-H. Hong, K. Ravi, A. Fallahi, G. Moriena, R. J. D. Miller, and F. X. Kärtner, "Terahertz-driven linear electron acceleration," Nature communications 6 (2015).

4. S. Carbajo, J. Schulte, X. Wu, K. Ravi, D. Schimpf, and F. Kärtner, "Efficient narrowband terahertz generation in cryogenically cooled periodically poled lithium niobate," Optics Letters 40, 5762-5765 (2015).

5. Z. Chen, X. Zhou, C. A. Werley, and K. A. Nelson, "Generation of high power tunable multicycle teraherz pulses," Applied Physics Letters 99, 071,102 (2011).

6. F. Ahr, S. Jolly, N. Matlis, S. Carbajo, T. Kroh, K. Ravi, D. Schimpf, J. Schulte, H. Ishizuki, T. Taira, and F. Kärtner, "Narrowband terahertz generation with chirped-and-delayed laser pulses in periodically poled lithium niobate," Optics Letters 42, 2118-2121 (2017).

7. K. Ravi, D. Schimpf, and F. Kärtner, "Pulse sequences for efficient multi-cycle terahertz generation in periodically poled lithium niobate," Optics Express 24, 5253-5276 (2016).

8. S. W. Jolly, "Spectral phase manipulation of optical pump pulses for mJ-level narrowband terahertz generation in PPLN," Ph.D. thesis, Universität Hamburg (2017).

9. H. Ishizuki and T. Taira, "Half-joule output optical-parametric oscillation using 10-mm-thick periodically poled Mg-doped congruent $\mathrm{LiNbO}_{3}$," Optics Express 20, 20,002-20,010 (2012). 